Article

\title{
Self-Similar Solutions of Rényi's Entropy and the Concavity of Its Entropy Power
}

\author{
Agapitos N. Hatzinikitas ${ }^{1,2}$ \\ ${ }^{1}$ Department of Mathematics, School of Sciences, University of Aegean, Karlovasi, Samos 83200, \\ Greece; E-Mail: ahatz@aegean.gr; Tel.: +30-22730-82131; Fax: +30-22730-82007 \\ ${ }^{2}$ School of Physics and Astronomy, University of Leeds, Leeds LS2 9JT, UK
}

Academic Editor: Demosthenes Ellinas

Received: 8 July 2015 / Accepted: 27 August 2015 / Published: 31 August 2015

\begin{abstract}
We study the class of self-similar probability density functions with finite mean and variance, which maximize Rényi's entropy. The investigation is restricted in the Schwartz space $S\left(\mathbb{R}^{d}\right)$ and in the space of l-differentiable compactly supported functions $C_{c}^{l}\left(\mathbb{R}^{d}\right)$. Interestingly, the solutions of this optimization problem do not coincide with the solutions of the usual porous medium equation with a Dirac point source, as occurs in the optimization of Shannon's entropy. We also study the concavity of the entropy power in $\mathbb{R}^{d}$ with respect to time using two different methods. The first one takes advantage of the solutions determined earlier, while the second one is based on a setting that could be used for Riemannian manifolds.
\end{abstract}

Keywords: maximum Rényi entropy; entropy power; Fisher information; nonlinear diffusion equation

MSC classifications: 94A17; 60E99

\section{Introduction}

The last two decades have witnessed an enormous growing interest in using information concepts in diverse fields of science. Although Rényi entropy was introduced as early as 1961, only recently, a wide range of applications has emerged as in the analysis of quantum entanglement [1], quantum correlations [2], computer vision [3], clustering [4], quantum cryptography [5] and pattern recognition [6,7]. 
Our contribution in this article is three-fold: First, we rigorously establish the solutions of two maximization problems ((12) and (33)) using self-similar probability density functions (pdfs) in the Schwartz space and the space of compactly-supported continuous functions on $\mathbb{R}^{d}$, by successfully applying the method of calculus of variations. Non-trivial mathematical technicalities, not addressed in previous maximization problems, are resolved, providing in this way a crystal clear presentation of the method, which was lacking from the literature. In particular, the perturbed pdfs we consider have the form $g_{\epsilon}=f+\epsilon h$, where $|\epsilon|<\epsilon_{0}<1$ and $h$ are functions, which are chosen in such a way that $g_{\epsilon}$ is a pdf and has the same variance as $f$. The vanishing of the first variation of the Lagrange functional provides us with an integral equation, which determines the critical points. It turns out that the solutions are unique. The non-negativity of the second variation leads to an integral inequality, which is preserved by the admissible perturbations we consider. Therefore, the critical point is a local maximum of the functional. To prove its global nature, we use the concept of the relative Rényi entropy and examine its positivity at the critical point. This procedure can be generalized on $\mathbb{R}^{d}$ straightforwardly. The solutions of the first optimization problem (12) have been derived in the past by [8], but following a completely different route. The second optimization problem (33), with the additional feature of non-zero mean, is proven to be equivalent to the first one by performing a suitable transformation to the random variable. Its solutions are new.

Second, we compare the specified solutions with those of Zel'dovich, Kompaneets and Barenblatt (ZKB) [9-11]. The difference is in the diffusion coefficient, which depends not only on the shape and the size of a molecule, but also on the order $\alpha$ of the Rényi entropy and the dimension of the space. We plot both our solution and the Barenblatt's solutions and observe that their $\mathcal{L}_{\infty}$ norms obey $\|u\|_{\infty} \leq\|f\|_{\infty}, \forall \frac{d}{d+2}<\alpha \leq \alpha_{\text {th. }}(d)$, while for values of $\alpha$ greater than the threshold $\alpha_{\text {th. }}(d)$, the inequality changes direction.

Third, the problem of concavity of the entropy power in $\mathbb{R}^{d}$ is confronted by utilising two different methods. In the first method, the solutions of the first problem guarantee concavity on the condition that the second time derivative of Rényi's entropy fulfils Inequality (49). The second method is closer to the spirit analysed in [12,13], where the $\alpha=1$ and $\alpha \neq 1$ cases were studied, respectively. The advantage of our approach stems from the simplicity of the arguments used in the proof and the discovery of the non-trivial Constraint (61), which is justified by self-consistency.

The paper is organized as follows. Section 2 reviews and proves some fundamental properties of the entropy. Sections 3,4 determine the solutions of the two maximization problems using the method of calculus of variations and examines their global validity using the concept of relative Rényi entropy. Section 5 constructs the nonlinear diffusion equation corresponding to the solutions of the two maximization problems and compares it to the usual one. Section 6 proves the concavity of the Rényi entropy with respect to time following two different methods. Section 7 concludes the work and comments on more general constraints one could have considered.

\section{Preliminaries}

In this section, we briefly review some properties of the Rényi entropy, and for the sake of clarity, we present the corresponding proofs. 
Definition 2.1. Let $(\Omega, \mathcal{A}, \mu)$ be a probability space and an $\mathcal{A}$-measurable function $f: \Omega \rightarrow \mathbb{R}^{+}$be a probability density function (pdf). The differential Rényi entropy of order $\alpha, \alpha \in \mathbb{R}^{+}$, is the nonlinear functional $H_{\alpha}: f \rightarrow \mathbb{R}$ defined by:

$$
H_{\alpha}[f]:=\frac{1}{1-\alpha} \ln \left(\int_{\Omega}\left(\frac{d F}{d \mu}\right)^{\alpha-1} d F\right)
$$

where $F$ is the probability measure induced by $f$, namely, $F(E)=\int_{E} f(x) d \mu(x), \forall E \in \mathcal{A}$.

Other equivalent ways of defining the Rényi entropy are: $H_{\alpha}[f]=\frac{1}{1-\alpha} \ln \left(\mathbb{E}_{X}\left(f^{\alpha-1}(X)\right)\right), \forall \alpha$ or $H_{\alpha}[f]=\frac{1}{1-\alpha} \ln \left(\|f\|_{L^{\alpha}}^{\alpha}\right), \forall \alpha>1$.

\section{Properties:}

(a) $H_{\alpha}[f]$ is a continuous $(\alpha \neq 1)$ and strictly decreasing function in $\alpha$ unless $f$ is the uniform density, in which case it is constant.

Proof: By Hölder's inequality, there is a family of relations:

$$
\int_{\Omega} f^{(1-\theta) p+\theta q} d \mu \leq\left(\int_{\Omega} f^{p} d \mu\right)^{1-\theta}\left(\int_{\Omega} f^{q} d \mu\right)^{\theta}
$$

holding whenever $p, q \geq 0$ and $\theta \in[0,1]$. Taking $p=1$ and assuming $f$ to be a pdf, we have:

$$
\int_{\Omega} f^{1-\theta+\theta q} d \mu \leq\left(\int_{\Omega} f^{q} d \mu\right)^{\theta} .
$$

Let $0<\alpha<\beta<1$ and $q=\alpha$. Then, for $\theta=(1-\beta) /(1-\alpha)<1$, the previous inequality becomes:

$$
\int_{\Omega} f^{\beta} d \mu \leq\left(\int_{\Omega} f^{\alpha} d \mu\right)^{\frac{1-\beta}{1-\alpha}}
$$

which, using that the logarithmic function is an increasing function, implies that $H_{\beta}[f] \leq H_{\alpha}[f]$. The same proof holds for $\alpha, \beta>1$.

(b) $H_{\alpha}(f)$ as a function of $\alpha$ converges to the following limits:

$$
\begin{aligned}
\lim _{\alpha \rightarrow 0^{+}} H_{\alpha}[f] & =\ln (\mu(\Omega)) \\
\lim _{\alpha \rightarrow 1} H_{\alpha}(f) & =H_{1}[f]=-\int_{\Omega} f \ln f d \mu \\
\lim _{\alpha \rightarrow \infty} H_{\alpha}[f] & =\|f\|_{\infty}=\inf \{0<\lambda<\infty: f \leq \lambda \text { a.e. } \omega \in \Omega\},
\end{aligned}
$$

where $H_{1}[f]$ is the Shannon entropy of $f$.

(c) If the $L_{1}\left(\Omega, \mathbb{R}^{+}\right)$norm is invariant under the homogeneous dilations:

$$
\begin{aligned}
x & \rightarrow \tilde{x}=\lambda^{\gamma} x, \quad \lambda>0, \forall x \in \Omega \subset \mathbb{R}^{d} \\
f(x) & \rightarrow f_{\lambda}(x)=\lambda^{\delta} f(\tilde{x})
\end{aligned}
$$


then $H_{\alpha}$, for $\alpha<1$, scales as:

$$
H_{\alpha}\left[f_{\lambda}\right]=H_{\alpha}[f]-\delta \ln \lambda, \quad \delta=d \gamma .
$$

Proof The $L_{1}$ norm invariance of $f$, namely $\|f\|_{L_{1}(\Omega)}=\|f\|_{L_{1}(\tilde{\Omega})}=M$, implies the condition $\delta=d \gamma$, which, combined with the definition of Rényi's entropy, produces the desired result.

\section{Formulation of the First Problem and Its Solutions}

In what follows, we restrict on the probability space $(\mathbb{R}, \mathcal{B}, d x)$, where $\mathcal{B}=\sigma(\mathcal{O})$ is the sigma algebra on open sets and $d x$ the Lebesgue measure on $\mathbb{R}$. The domain of the Rényi functional, $\mathcal{D}\left(H_{\alpha}\right)$, is defined to be:

$$
\begin{aligned}
& \mathcal{D}\left(H_{\alpha<1}\right)=\left\{f \in S(\mathbb{R}): \int_{\mathbb{R}} f(x) d x=1\right\} \\
& \mathcal{D}\left(H_{\alpha>1}\right)=\left\{f_{+} \in C_{c}^{k}(\mathbb{R}), k \in \mathbb{Z}^{+} \text {and } k \leq\left[\frac{1}{\alpha-1}\right]: \int_{\mathbb{R}} f_{+}(x) d x=1\right\},
\end{aligned}
$$

where $f$ is a positive and integrable real-valued function, $f_{+}(x)=f(x) \chi_{B}(x)$ with $\chi$ the indicator function of the set $B$, and [.] is the integer part of the number.

The first entropy maximization problem with vanishing mean and finite variance is formulated as:

$$
\underset{f \in \mathcal{D}\left(H_{\alpha<1}\right)}{\max . H_{\alpha}[f]} \quad \text { subjected to the constraint } \quad \mathbb{E}\left(X^{2}\right)=\int_{\mathbb{R}} x^{2} f(x) d x=\mu_{2}^{2}<\infty .
$$

Using the method of Lagrange multipliers, we construct the functional:

$$
\mathcal{F}\left(f ; \lambda_{0}, \lambda_{2}\right)=H_{\alpha}[f]-\sum_{k \in\{0,2\}} \lambda_{k}\left(\mathbb{E}\left(X^{k}\right)-\mu_{k}^{2}\right), \quad \mu_{0}=1
$$

and impose appropriate conditions on the perturbations in order to calculate its first and second variation.

Definition 3.1. A perturbation $h \in S(\mathbb{R})$ is called admissible if it satisfies the following conditions:

$$
|h(x)|<c|f(x)|, 0<c<1, \forall x \in \mathbb{R}, \int_{\mathbb{R}} h(x) d x=0 \text { and } \int_{\mathbb{R}} x^{2} h(x) d x=0 .
$$

If we introduce the usual inner product in $S(\mathbb{R})$, the previous integral conditions imply that we search for a class of functions, which are orthogonal to the unity and $x^{2}$. Odd functions in the Schwartz space, such as $Q(x) e^{-b|x|^{\mu}}, b, \mu>0$, with $Q(x)$ a polynomial of odd powers of $x$, satisfy these criteria.

Expanding the Lagrange functional in a Taylor series up to second order in $\epsilon$, we obtain:

$$
\mathcal{F}\left(f+\epsilon h ; \lambda_{0}, \lambda_{2}\right)=\mathcal{F}\left(f ; \lambda_{0}, \lambda_{2}\right)+\delta \mathcal{F}_{f}(h) \epsilon+\delta^{2} \mathcal{F}_{f}(h) \epsilon^{2}+o\left(\epsilon^{2}\right) .
$$

The first-order necessary condition for optimality requires [14]:

$$
\delta \mathcal{F}_{\hat{f}}(h)=\left.\frac{d}{d \epsilon} \mathcal{F}\left(f+\epsilon h ; \lambda_{0}, \lambda_{2}\right)\right|_{\epsilon=0}=0, \quad|\epsilon|<\epsilon_{0}<1
$$

or, equivalently,

$$
\int_{\mathbb{R}}\left(f^{\alpha-1}(x)-\sum_{k \in\{0,2\}} \tilde{\lambda}_{k} x^{k}\right) h(x) d x=0, \quad \text { where } \quad \tilde{\lambda}_{k}=\lambda_{k} g(\alpha)=\lambda_{k} \frac{(1-\alpha)}{\alpha} \int_{\mathbb{R}} f^{\alpha}(x) d x .
$$

The function $f(x)$ is determined by using the following lemma. 
Lemma 3.2. If $f \in S(\mathbb{R})$ and if:

$$
\int_{\mathbb{R}} f(x) g(x) d x=0, \forall g \in S(\mathbb{R}) \quad \text { such that } \quad \lim _{|x| \rightarrow \infty} g(x)=0
$$

then $f(x)=0, \forall x \in \mathbb{R}$.

Proof Suppose that $f(x) \neq 0$, then there exists $\xi \in \mathbb{R}$, such that $f(\xi)=c>0$ (assuming that the constant is positive). Since $f \in S(\mathbb{R})$, there exists a neighbourhood $(a, b)$ of $\xi$ in which $f(x)>0$, $\forall x \in(a, b)$. Define the function:

$$
g(x)=(x-a)(b-x) e^{-x^{2}} \chi_{(a, b)}(x)
$$

The function $g(x)$ is positive in $(a, b)$ with $g(a)=g(b)=0$. However:

$$
\int_{\mathbb{R}} f(x) g(x) d x=\int_{a}^{b} f(x)(x-a)(b-x) e^{-x^{2}} d x>0
$$

since the integrand is positive (except at $\mathrm{a}$ and $\mathrm{b}$ ). This contradiction proves the lemma.

Therefore, $f(x)$ is given by:

$$
\hat{f}(x)=\left(\sum_{k \in\{0,2\}} \tilde{\lambda}_{k} x^{k}\right)^{-\frac{1}{1-\alpha}}=\|\hat{f}\|_{\infty}\left(1+\frac{\tilde{\lambda}_{2}}{\tilde{\lambda}_{0}} x^{2}\right)^{-\frac{1}{1-\alpha}},
$$

where the $L^{\infty}$ norm is with respect to $\mathrm{x}$ and $\tilde{\lambda}_{k}>0$, since $\hat{f} \in \mathcal{D}_{\alpha<1}$. In order for $\hat{f}$ to be a local maximum, the following second-order necessary condition for optimality should also hold:

$$
\left.\delta^{2} \mathcal{F}\right|_{\hat{f}}(h) \leq 0 \quad \text { or }\left.\quad \frac{d^{2}}{d \epsilon^{2}} \mathcal{F}\left(f+\epsilon h ; \lambda_{0}, \lambda_{2}\right)\right|_{\epsilon=0, f=\hat{f}} \leq 0 .
$$

In other words, the second variation of $\mathcal{F}$ at $\hat{f}$ should be positive semidefinite on the space of admissible perturbations $h$. The previous inequality is translated into:

$$
\frac{\alpha}{\alpha-1} \int_{\mathbb{R}} \hat{f}^{\alpha-1}(x) h(x) d x \leq\left(\int_{\mathbb{R}} \hat{f}^{\alpha}(x) d x\right)\left(\int_{\mathbb{R}} \hat{f}^{\alpha-2}(x) h^{2}(x) d x\right) .
$$

It is easily checked that the solution $\hat{f}$ together with the admissible perturbations satisfy the strict inequality; therefore, $\hat{f}$ is a strict one-parameter family of local maxima with increasing Rényi entropy, as Property (a) of Section 2 guarantees.

Remark If $0<\int_{\mathbb{R}} f^{\alpha}(x) d x \leq 2$, then Taylor expanding the logarithmic function around unity, the Rényi entropy reduces to the Havrda-Charvát entropy [15], also called Tsallis entropy [16],

$$
S_{\alpha}(f)=\frac{1}{\alpha-1}\left(1-\int_{\mathbb{R}} f^{\alpha}(x) d x\right) .
$$

The solution in this case is the previous one, but with the substitution $\tilde{\lambda}_{k}=\lambda_{k} \frac{(1-\alpha)}{\alpha}$.

Depending on the space of functions, we distinguish the following two types of solutions. 
$(3 \alpha)$ The $\boldsymbol{S}(\mathbb{R})$ solution $(\boldsymbol{\alpha}<\mathbf{1})$. The requirement that $\hat{f}$ is a pdf and the second moment constraint lead to the relation:

$$
\frac{\tilde{\lambda}_{0}}{\tilde{\lambda}_{2}} \frac{B_{\frac{3}{2}}}{B_{\frac{1}{2}}}=\frac{\lambda_{0}}{\lambda_{2}}\left(\frac{1-\alpha}{3 \alpha-1}\right)=\mu_{2}^{2} \quad \text { for } \quad \frac{1}{3}<\alpha<1 .
$$

We adopt the abbreviation $B_{s / 2} \equiv B(s / 2,1 /(1-\alpha)-s / 2)$ for Euler's beta function defined by $B(x, y)=\int_{0}^{1} t^{x-1}(1-t)^{y-1} d t$ for $\operatorname{Re}(x), \operatorname{Re}(y)>0$. Using (25), the exact solution can be expressed as:

$$
\hat{f}(x)=\left(\frac{1-\alpha}{3 \alpha-1}\right)^{\frac{1}{2}} \frac{1}{\mu_{2} B\left(\frac{1}{2}, \frac{1}{1-\alpha}-\frac{1}{2}\right)}\left(1+\left(\frac{1-\alpha}{3 \alpha-1}\right) \frac{x^{2}}{\mu_{2}^{2}}\right)^{-\frac{1}{1-\alpha}}, \quad \frac{1}{3}<\alpha<1 .
$$

This one-parameter family of local maxima of $H_{\alpha}$ is unique, and it remains to prove that it is actually also a one-parameter family of global maxima in $\mathcal{D}\left(H_{\alpha<1}\right)$. For this, we use the notion of the relative $\alpha$-Rényi entropy of two densities $\hat{f}$ and $g$, defined in [17] by:

$$
D_{\alpha}(g \| \hat{f})=\frac{1}{1-\alpha} \ln \left(\int_{\mathbb{R}} \hat{f}^{\alpha-1}(x) g(x) d x\right)+\frac{(1-\alpha)}{\alpha} H_{\alpha}[\hat{f}]-\frac{1}{\alpha} H_{\alpha}[g],
$$

where $g$ satisfies the same second moment constraint as $\hat{f}$. The first term in the right-hand side of (27) equals $H_{\alpha}[\hat{f}]$, as one may check, since $\int_{\mathbb{R}} \hat{f}^{\alpha-1}(x) g(x) d x=\int_{\mathbb{R}} \hat{f}^{\alpha}(x) d x$. Therefore:

$$
D_{\alpha}(g \| \hat{f})=\frac{1}{\alpha}\left(H_{\alpha}[\hat{f}]-H_{\alpha}[g]\right) \geq 0
$$

by applying Hölder's inequality to the functions $\left(\hat{f}^{\alpha-1} g\right)^{\alpha}$ and $\hat{f}^{\alpha(\alpha-1)}$. The same result holds in the $\alpha>1$ case.

Proposition 3.3. The optimization problem for $\alpha<1$ has a one-parameter family of global maxima $\hat{f}$, which in the $\alpha \rightarrow 1^{-}$limit converges to the global maximum of the Shannon entropy, which is the normal distribution $\mathcal{N}\left(0, \mu_{2}\right)$.

Proof Relation (26) in the $\alpha \rightarrow 1^{-}$limit gives:

$$
\lim _{\alpha \rightarrow 1^{-}} \tilde{\lambda}_{0}^{-\frac{1}{1-\alpha}}(\alpha)\left(1+\left(\frac{1-\alpha}{3 \alpha-1}\right) \frac{x^{2}}{\mu_{2}^{2}}\right)^{-\frac{1}{1-\alpha}}=\frac{1}{\sqrt{2 \pi \mu_{2}^{2}}} \lim _{\rho \rightarrow 0^{+}}\left(1+\frac{x^{2}}{2 \mu_{2}^{2}} \rho\right)^{-\frac{1}{\rho}}=\frac{1}{\sqrt{2 \pi \mu_{2}^{2}}} e^{-\frac{x^{2}}{2 \mu_{2}^{2}}}
$$

where the change of variable $\rho=(3 \alpha-1) / 2(1-\alpha)$ has been performed.

(3ß) The 1-differentiable compactly-supported solution $(\boldsymbol{\alpha}>\mathbf{1})$. In this case, following steps similar to the previous one, the solution turns out to be:

$$
\hat{f}(x)=\tilde{\lambda}_{0}^{\frac{1}{\alpha-1}}\left(1-\left(\frac{\alpha-1}{3 \alpha-1}\right) \frac{x^{2}}{\mu_{2}^{2}}\right)_{+}^{\frac{1}{\alpha-1}}, \alpha>1 \quad \text { and } \quad x_{+} \equiv \max \{0, x\} \equiv x \theta(x),
$$

where $\theta(x)$ is the unit-step function and the arbitrary constant $\tilde{\lambda}_{0}$ is specified as usual by imposing the requirement that $\hat{f}$ be a pdf:

$$
\tilde{\lambda_{0}}(\alpha)=\left(\left(\frac{\alpha-1}{3 \alpha-1}\right)^{\frac{1}{2}} \frac{1}{\mu_{2} B\left(\frac{1}{2}, \frac{1}{\alpha-1}+1\right)}\right)^{\alpha-1}
$$


Remarks The derivations of $(3 \alpha)$ and $(3 \beta)$ are based on the integral Formulas (3.241.4) and (3.251.1) of [18], which are connected through the transformation $u=x^{2} /\left(x^{2}+1\right)$ with $u \in(0,1)$; see (A.2). The solution $(3 \alpha)$ can also be derived by integrating Equation (17), in which case, we obtain the relation between the Lagrange multipliers:

$$
\lambda_{0}=\frac{\alpha}{1-\alpha}-\mu_{2}^{2} \lambda_{2}
$$

Eliminating $\lambda_{0}$ from the solution and applying the constraints, we arrive at (26).

The previous setup can be generalized to include the case of a covariance matrix $C$-constraint. The new solution can be derived from the old one by replacing $x^{2}$ by $x^{T} C^{-1} x$; see [8].

\section{Formulation of the Second Problem and Its Solutions}

The second entropy maximization problem, with non-vanishing mean and finite variance, can equivalently be restated as:

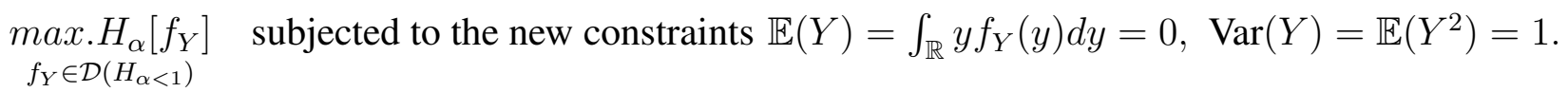

The random variables $X, Y$ are related through the transformation $X=\mu_{2} Y+\mu_{1}$, and their corresponding pdfs $f_{X}, f_{Y}$ satisfy $f_{X}(x)=f_{Y}\left(\left(x-\mu_{1}\right) / \mu_{2}\right) / \mu_{2}$. As a consequence, the entropies are connected through:

$$
H_{\alpha}\left[f_{X}\right]=H_{\alpha}\left[f_{Y}\right]+\ln \mu_{2}
$$

The solutions of the second problem (33) are therefore given by the solutions of the first problem (12), with the substitution $\left(x-\mu_{1}\right) / \mu_{2} \rightarrow x$. The first-order necessary optimization condition dictates the solution:

$$
\hat{f}^{\alpha-1}(x)=\sum_{k=0}^{2} \tilde{\lambda}_{k} x^{k}
$$

which can also be proven to be a global maximum.

We distinguish the following two classes of solutions.

$(4 \alpha)$ The $\boldsymbol{S}(\mathbb{R})$ solution $(\boldsymbol{\alpha}<\mathbf{1})$. The positivity of the solution $\hat{f}^{\alpha-1}(x), \forall x \in \mathbb{R}$ requires $\tilde{\lambda}_{2}>0$ and $\tilde{\lambda}_{2} \tilde{\lambda}_{0}-\left(\tilde{\lambda}_{1}\right)^{2}>0$. The pdf, the mean value and the variance constraints lead to the conditions:

$$
\mu_{1}=-\frac{\lambda_{1}}{\lambda_{2}}, \quad \frac{\lambda_{0}}{\lambda_{2}}=\frac{(2 n-3) ! !}{(2 n-5) ! !} \mu_{2}^{2}+\mu_{1}^{2}, \quad n=\frac{1}{1-\alpha} \in \mathbb{Z}^{+} .
$$

Finally, the solution is written as:

$$
\hat{f}(x)=\frac{(2 n-2) ! !}{(2 n-3) ! !} \sqrt{\frac{(2 n-5) ! !}{(2 n-3) ! !}} \frac{1}{\mu_{2}}\left(1+\frac{(2 n-3) ! !}{(2 n-5) ! !}\left(\frac{x-\mu_{1}}{\mu_{2}}\right)^{2}\right)^{-n}
$$

which is identical to the solution derived from the equivalent problem. 
(4ß) The 1-differentiable compactly-supported solution $(\boldsymbol{\alpha}>\mathbf{1})$. In this case, the polynomial $\sum_{k} \tilde{\lambda}_{k} x^{k}$ should be positive between its real roots. This occurs provided that $\tilde{\lambda}_{2}<0$ and $\left(\tilde{\lambda}_{1}\right)^{2}-\tilde{\lambda}_{2} \tilde{\lambda}_{0}>0$. Using the indicator function $\chi_{\left(x_{1}, x_{2}\right)}$, with $x_{1}, x_{2}$ the roots of the polynomial, we find the previous solution with a relative minus sign between the terms inside the parentheses, while the power is now positive.

\section{Comparison with the FDE and PME Solutions}

The pdf, $\hat{f}$, which maximizes the Shannon entropy under the finiteness of the second moment turns out to be identical to the fundamental solution of the diffusion equation with a Dirac point source. It is worth noting that $\hat{f}$ is actually a global maximum of $H_{1}$. This observation is accidental, as one may justify from the study of the corresponding optimization problem for the Rényi entropy. In particular, the nonlinear, initial-valued problem:

$$
\begin{aligned}
\partial_{t} u(x, t) & =\Delta u^{\alpha}(x, t), \quad(x, t) \in \mathbb{R}^{d} \times(0, \infty) \\
\lim _{t \rightarrow 0^{+}}\left(\int_{\mathbb{R}^{d}} u(x, t) d x\right) & =\delta_{0}(x)
\end{aligned}
$$

has the following self-similar solutions [19]:

$$
\begin{aligned}
& \text { FDE solution: } u(x, t)_{\alpha<1}=t^{-\delta}\left(C_{\alpha<1}+\frac{(1-\alpha) \delta}{2 \alpha d}\left(\frac{x}{t^{\gamma}}\right)^{2}\right)^{-\frac{1}{1-\alpha}}, \\
& \text { PME solution: } u(x, t)_{\alpha>1}=t^{-\delta}\left(C_{\alpha>1}-\frac{(\alpha-1) \delta}{2 \alpha d}\left(\frac{x}{t^{\gamma}}\right)^{2}\right)_{+}^{\frac{1}{\alpha-1}},
\end{aligned}
$$

where FDE, PME stand for fast diffusion equation, porous medium equation, respectively,

$$
\begin{aligned}
\delta & =d \gamma, \quad \gamma=\frac{1}{(2+d(\alpha-1))}, \quad|\kappa|=\frac{|1-\alpha|}{2 \alpha d} \delta \\
C_{\alpha<1} & =\left(\frac{B\left(\frac{d}{2}, \frac{1}{1-\alpha}-\frac{d}{2}\right)\left|S_{d-1}\right|}{2|\kappa|^{\frac{d}{2}}}\right)^{2 \gamma(1-\alpha)} \text { and } C_{\alpha>1}=\left(\frac{2|\kappa|^{\frac{d}{2}}}{B\left(\frac{d}{2}, \frac{\alpha}{\alpha-1}\right)\left|S_{d-1}\right|}\right)^{2 \gamma(\alpha-1)} .
\end{aligned}
$$

The $d$-dimensional time-dependent functions:

$$
\begin{aligned}
& f(x, t)_{\alpha<1}= \frac{1}{\mu_{2}^{d}(t)} A_{\alpha<1}\left(1+\beta \frac{x^{2}}{\mu_{2}^{2}(t)}\right)^{-\frac{1}{1-\alpha}} \quad \text { where } \beta \equiv \beta(\alpha, d)=\frac{|1-\alpha|}{(2 \alpha-d(1-\alpha))}, \\
& A_{\alpha<1}=\beta^{\frac{d}{2}} \frac{2}{\left|\mathbb{S}_{d-1}\right| B\left(\frac{d}{2}, \frac{1}{1-\alpha}-\frac{d}{2}\right)} \quad \text { if } \quad \frac{d}{d+2}<\alpha<1 \text {, and } \\
& f(x, t)_{\alpha>1}= \frac{1}{\mu_{2}^{d}(t)} A_{\alpha>1}\left(1-\beta \frac{x^{2}}{\mu_{2}^{2}(t)}\right)_{+}^{\frac{1}{\alpha-1}} \\
& \text { where } A_{\alpha>1}=\beta^{\frac{d}{2}} \frac{2}{\left|\mathbb{S}_{d-1}\right| B\left(\frac{d}{2}, \frac{\alpha}{\alpha-1}\right)} \text { if } \alpha>1
\end{aligned}
$$

derived from the optimization of Rényi's entropy, can be shown to satisfy the following initial value problem:

$$
\begin{aligned}
K_{\alpha} \partial_{t} f(x, t) & =\Delta f^{\alpha}(x, t),(x, t) \in \mathbb{R}^{d} \times(0, \infty) \\
\lim _{t \rightarrow 0^{+}}\left(\int_{\mathbb{R}^{d}} f(x, t) d x\right) & =1
\end{aligned}
$$


provided that $\gamma \equiv \gamma(\alpha, d)=1 /(2+d(\alpha-1))$, and the coefficient is given by:

$$
K_{\alpha \lessgtr 1}=\frac{2 \alpha(2+d(\alpha-1))}{(2 \alpha+d(\alpha-1))} A_{\alpha \lessgtr 1}^{\alpha-1},
$$

where $\mu_{2}(t)=t^{\gamma}$. The presence of the ratio $x / t^{\gamma}$ is implied by the self-similar property of the solution, which requires the function in the parentheses to remain invariant under the rescalings: $x \rightarrow \tilde{x}=\lambda^{\gamma} x$ and $t \rightarrow \tilde{t}=\lambda t$. Therefore, in general, the pdf maximizing the Rényi entropy is a solution of an appropriately-constructed diffusion equation problem.

In Figures 1 and 2, we plot the FDE and PME solutions of (38) versus the solutions of (45), respectively.

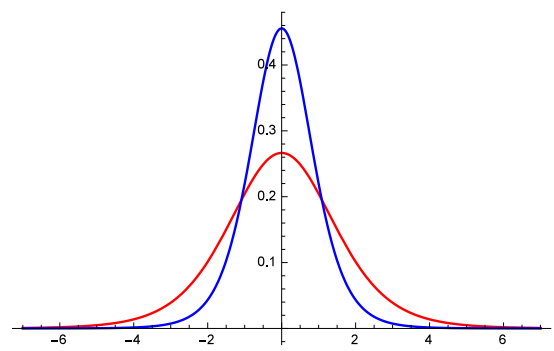

Figure 1. A snapshot at $t=1$ of the FDE solution of (38) (red line) and (45) (blue line) initial-value problems, with parameters: $d=1, \alpha=3 / 4$.

In the fast diffusion case $\|u\|_{\infty}<\|f\|_{\infty}$, since $C_{\alpha<1}<A_{\alpha<1}, \forall \frac{d}{d+2}<\alpha<1$, as one may prove using Proposition (7.1) of the Appendix.

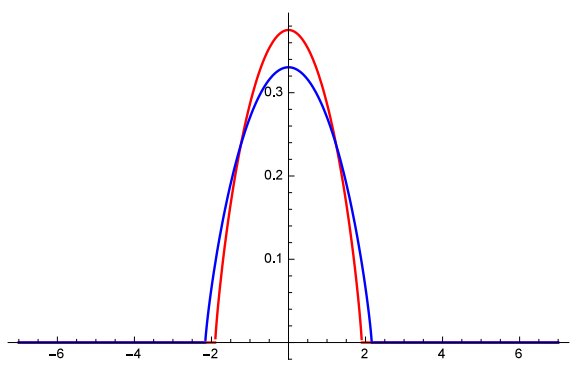

Figure 2. A snapshot at $t=1$ of the PME solution of (38) (red line) and (45) (blue line) initial-value problems, with parameters: $d=1, \alpha=2.2$.

In the porous medium regime $\|u\|_{\infty}<\|f\|_{\infty}$ up to the threshold value $\alpha_{\text {th. }}(d=1)=1.8268$, for which $C_{\alpha>1}=A_{\alpha>1}$ and then $\|u\|_{\infty}>\|f\|_{\infty}, \forall \alpha>\alpha_{\text {th. }}$. The threshold value $\alpha_{\text {th. }}$, which depends on the dimension $d$, is determined arithmetically.

\section{The Concavity of Rényi’s Entropy Power}

Definition 6.1. Let $f: \Omega \times(0, \infty) \subset \mathbb{R}^{d} \times(0, \infty) \rightarrow \mathbb{R}_{+}^{d} \times(0, \infty)$ be a pdf in $\mathcal{D}_{\alpha}$. The $\alpha$-weighted Fisher information of $f$ is defined by:

$$
I_{\alpha}[f](t)=\frac{1}{\int_{\Omega} f^{\alpha} d \mu} \int_{\Omega} \frac{\left|\nabla f^{\alpha}\right|^{2}}{f} d \mu,
$$


while the entropy power of $f$ associated with the Rényi entropy $H_{\alpha}$ is defined by:

$$
\mathcal{N}_{\alpha}[f](t)=\left\{\begin{array}{lc}
e^{\left(\frac{2}{d}+(\alpha-1)\right) H_{\alpha}[f](t)}=\left(\int_{\Omega} f^{\alpha} d \mu\right)^{-1}\left(\int_{\Omega} f^{\alpha} d \mu\right)^{\frac{2}{d(1-\alpha)}}, & 0<\alpha<\infty, \quad \alpha \neq 1 \\
e^{\frac{2}{d} H_{1}[f](t)}, & \alpha=1 \\
\mu(\{x: f(x, t)>0\})^{\frac{2}{d}-1}, & \alpha=0 .
\end{array}\right.
$$

Proposition 6.2. Let $\Omega=\mathbb{E}^{d}$ be the d-dimensional Euclidean space. The entropy power $\mathcal{N}_{\alpha}$ is a concave function of $t, \forall t \in(0, \infty)$ provided that:

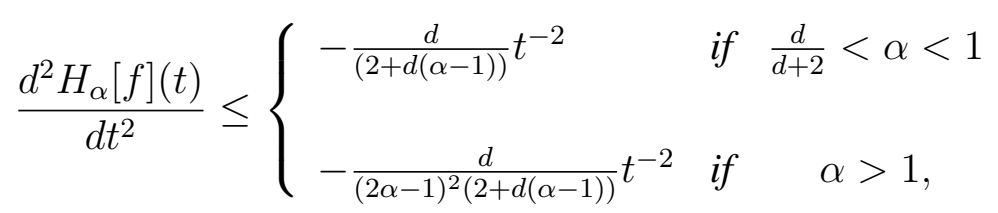

where the right-hand side of the inequality represents the contributions from the global maximum of $H_{\alpha}$. Proof Using the nonlinear diffusion equation, a straightforward calculation reveals the connection between $d H_{\alpha} / d t$ and $I_{\alpha}$ expressed by the relation:

$$
\frac{d H_{\alpha}[f](t)}{d t}=K_{\alpha}^{-1} I_{\alpha}[f](t)
$$

The entropy power is a concave function of time iff:

$$
\frac{d^{2} \mathcal{N}_{\alpha}[f](t)}{d t^{2}} \leq 0 \Longleftrightarrow \frac{d^{2} H_{\alpha}[f](t)}{d t^{2}}=K_{\alpha}^{-1} \frac{\left.d I_{\alpha}[f](t)\right)}{d t} \leq-\left(\frac{2}{d}+(\alpha-1)\right)\left(\frac{d H_{\alpha}[f](t)}{d t}\right)^{2} .
$$

The first step is to establish the identity:

$$
\frac{d H_{\alpha}[f](t)}{d t}=\frac{\alpha^{2} K_{\alpha}^{-1}}{(1-2 \alpha) \int_{\mathbb{R}^{d}} f^{\alpha} d x} \int_{\mathbb{R}^{d}} f^{2 \alpha-1} \Delta(\ln f) d x .
$$

For this reason, we rewrite the integral as:

$$
\begin{aligned}
\int_{\mathbb{R}^{d}} \frac{\left|\nabla f^{\alpha}\right|^{2}}{f} d x & =\frac{\alpha(1-\alpha)}{(2 \alpha-1)} \int_{\mathbb{R}^{d}} \nabla f^{2 \alpha-1} \cdot \nabla(\ln f) d x-\alpha \int_{\mathbb{R}^{d}} f^{2 \alpha-1} \Delta(\ln f) d x \\
& =\frac{\alpha^{2}}{(1-2 \alpha)} \int_{\mathbb{R}^{d}} f^{2 \alpha-1} \Delta(\ln f) d x .
\end{aligned}
$$

The next step is to prove that the first time derivative of the Rényi entropy satisfies the following upper bounds:

$$
\frac{d H_{\alpha}[f](t)}{d t} \leq \begin{cases}\frac{d}{(2+d(\alpha-1))} t^{-1} & \text { if } \quad \frac{d}{d+2}<\alpha<1 \\ \frac{d}{(2 \alpha-1)(2+d(\alpha-1))} t^{-1} & \text { if } \quad \alpha>1\end{cases}
$$

Note that the contribution of the integral involving the term $\Delta(\ln \hat{f})$ is given by:

$$
\begin{aligned}
\int_{\mathbb{R}^{d}} \hat{f}^{2 \alpha-1} \Delta(\ln \hat{f}) d x= & -\frac{1}{|1-\alpha|} \frac{2 \beta^{1-\frac{d}{2}}\left|\mathbb{S}_{d-1}\right|}{t^{2 \gamma(1+d(\alpha-1))}} \\
& \times\left\{\begin{array}{l}
A_{\alpha<1}^{2 \alpha-1}\left[d B\left(\frac{d}{2}, \frac{1}{1-\alpha}-\frac{d}{2}\right)+(d-2) B\left(1+\frac{d}{2}, \frac{\alpha}{1-\alpha}-\frac{d}{2}\right)\right] \text { if } \frac{d}{d+2}<\alpha<1 \\
A_{\alpha>1}^{2 \alpha-1}\left[d B\left(\frac{d}{2}, \frac{\alpha}{\alpha-1}\right)-(d+2) B\left(1+\frac{d}{2}, \frac{\alpha}{\alpha-1}+1\right)\right] \quad \text { if } \quad \alpha>1,
\end{array}\right.
\end{aligned}
$$


while:

$$
\frac{\alpha^{2}}{(1-2 \alpha)} \frac{K_{\alpha}^{-1}}{\int_{\mathbb{R}^{d}} f^{\alpha}(x, t) d x}=\frac{\alpha(2 \alpha+d(\alpha-1)) \beta^{\frac{d}{2}} t^{d \gamma(\alpha-1)}}{2(1-2 \alpha)(2+d(\alpha-1))\left|\mathbb{S}_{d-1}\right|}\left\{\begin{array}{ccc}
\frac{A_{\alpha<1}^{1-2 \alpha}}{B\left(\frac{d}{2}, \frac{\alpha}{1-\alpha}-\frac{d}{2}\right)} & \text { if } \frac{d}{d+2}<\alpha<1 \\
\frac{A_{\alpha>1}^{1-2 \alpha}}{B\left(\frac{d}{2}, \frac{\alpha}{\alpha-1}+1\right)} & \text { if } \quad \alpha>1 .
\end{array}\right.
$$

Substituting (54) into (51), we recover the expected result. Note that in the $\alpha \rightarrow 1^{-}$limit, we reproduce the well-known result valid for Shannon's entropy.

Next, we prove the concavity of Rény's power entropy on a different setting. This problem was also studied in [13], but our approach leads to a condition not predicted before. We will need the following lemma.

Lemma 6.3. Let $G$ be defined as:

$$
G_{\alpha}[f](t)=\int_{\mathbb{R}^{d}} \frac{\left|\nabla f^{\alpha}\right|^{2}}{f} d x=\int_{\mathbb{R}^{d}} f|\nabla v|^{2} d x \quad \text { with } \quad v=\frac{\alpha}{\alpha-1} f^{\alpha-1}
$$

then:

$$
\begin{aligned}
\frac{d G_{\alpha}[f](t)}{d t} & =-2 K_{\alpha}^{-1} \int_{\mathbb{R}^{d}} f^{\alpha}\left(\|\nabla \nabla v\|^{2}+(\alpha-1)(\Delta v)^{2}\right) d x \quad \text { and } \\
\frac{d I_{\alpha}[f](t)}{d t} & =(1-\alpha) K_{\alpha}^{-1} I_{\alpha}^{2}+\frac{1}{\int_{\mathbb{R}^{d}} f^{\alpha} d x} \frac{d G_{\alpha}[f](t)}{d t}
\end{aligned}
$$

Proof Using the porous medium equations for $f, v$, as well as the relation $\nabla f^{\alpha}=f \nabla v$, we have:

$$
\begin{aligned}
\frac{d G[f](t)}{d t} & =\int_{\mathbb{R}^{d}}\left(f_{t}|\nabla v|^{2}+2 f_{t} \nabla v(x, t) \cdot \nabla v_{t}\right) d x, v_{t}=\frac{\partial v}{\partial t} \\
& =K_{\alpha}^{-1} \int_{\mathbb{R}^{d}}\left(\Delta f^{\alpha}|\nabla v|^{2}+2 f \nabla v \cdot \nabla\left((\alpha-1) v \nabla v+|\nabla v|^{2}\right)\right) d x \\
& =K_{\alpha}^{-1} \int_{\mathbb{R}^{d}}\left(\Delta f^{\alpha}|\nabla v|^{2}+2(\alpha-1)\left(f|\nabla v|^{2} \Delta v+f v \nabla v \cdot \nabla \Delta v\right)+2 f \nabla v \cdot \nabla|\nabla v|^{2}\right) d x \\
& =K_{\alpha}^{-1} \int_{\mathbb{R}^{d}}\left(\Delta f^{\alpha}|\nabla v|^{2}+2(\alpha-1) \nabla f^{\alpha} \cdot \nabla v \Delta v+2 \alpha f^{\alpha} \nabla v \cdot \nabla \Delta v+2 \nabla f^{\alpha} \cdot \nabla|\nabla v|^{2}\right) d x \\
& =K_{\alpha}^{-1} \int_{\mathbb{R}^{d}}\left(\left(\Delta f^{\alpha}-2 \Delta f^{\alpha}\right)|\nabla v|^{2}-2(\alpha-1) f^{\alpha}\left((\Delta v)^{2}+\nabla v \cdot \nabla \Delta v\right)+2 \alpha f^{\alpha} \nabla v \cdot \nabla \Delta v\right) d x \\
& =K_{\alpha}^{-1} \int_{\mathbb{R}^{d}}\left(f^{\alpha}\left(2 \nabla v \cdot \nabla \Delta v-\Delta\left(|\nabla v|^{2}\right)\right)-2(\alpha-1) f^{\alpha}(\Delta v)^{2}\right) d x \\
& =-2 K_{\alpha}^{-1} \int_{\mathbb{R}^{d}} f^{\alpha}\left(\|\nabla \nabla v\|^{2}+(\alpha-1)(\Delta v)^{2}\right) d x
\end{aligned}
$$

where partial integrations in the fourth and fifth equalities have been used. Furthermore, in the last step, Bochner's formula in Euclidean space has been applied. Relation (59) is proven using (58).

Theorem 6.4. The Rényi entropy power, for self-similar solutions, is concave in $t$, provided that the following inequality is satisfied:

$$
\int_{\mathbb{R}^{d}}\left(f^{\alpha}(\xi)-C f^{2 \alpha-1}(\xi)\right) d \xi \geq 0, \quad \xi=\frac{x}{t^{\gamma}}, \quad C=\frac{1}{\alpha^{2}(1+d(\alpha-1))},
$$

where the equality holds for $\alpha=1$. 
Proof The Rényi entropy power is concave in t iff Condition (51) holds, which can be written equivalently as:

$$
\begin{aligned}
I_{\alpha}^{2}[f](t) & \leq d\left(\int_{\mathbb{R}^{d}} f^{\alpha} d x\right)^{-1} \int_{\mathbb{R}^{d}} f^{\alpha}\left(\|\nabla \nabla v\|^{2}+(\alpha-1)(\Delta v)^{2}\right) d x \\
& \leq d(1+d(\alpha-1))\left(\int_{\mathbb{R}^{d}} f^{\alpha} d x\right)^{-1} \int_{\mathbb{R}^{d}} f^{\alpha}\|\nabla \nabla v\|^{2} d x \\
& \leq d(1+d(\alpha-1)) J_{\alpha}[f](t),
\end{aligned}
$$

where $\|\nabla \nabla v\|^{2}=\|\operatorname{Hess} v\|^{2}=\sum_{i, j=1}^{d}\left(\partial_{i j} v\right)^{2}$, and we used the identity $\|\operatorname{Hess} v\|^{2}=\operatorname{Tr}\left((\operatorname{Hess} v)^{2}\right) \geq$ $(\operatorname{Tr}(\operatorname{Hess} v))^{2} / d$. Expanding $\|\cdot\|^{2}$ and writing it in terms of the logarithm of $f$, the last integral can be cast into the form:

$$
\int_{\mathbb{R}^{d}} f^{\alpha}\|\nabla \nabla v\|^{2} d x=\alpha^{2} \int_{\mathbb{R}^{d}} f^{2 \alpha-1} \sum_{i, j=1}^{d}\left((\alpha-1)\left(\partial_{i} \ln f\right)\left(\partial_{j} \ln f\right)+\partial_{i j}^{2} \ln f\right)^{2} d x,
$$

which by applying the Cauchy-Schwarz inequality, we get that:

$$
\int_{\mathbb{R}^{d}} f^{\alpha}\|\nabla \nabla v\|^{2} d x \geq \frac{\alpha^{2}}{d}\left(\int_{\mathbb{R}^{d}} f^{2 \alpha-1} d x\right)^{-1}\left(\int_{\mathbb{R}^{d}} f^{2 \alpha-1}\left((\alpha-1)|\nabla \ln f|^{2}+\Delta \ln f\right) d x\right)^{2} .
$$

Using the identity $\frac{\Delta f^{\alpha}}{f^{\alpha}}=\left|\nabla \ln f^{\alpha}\right|^{2}+\Delta \ln f^{\alpha}$, we eliminate the $|\nabla \ln f|^{2}$ term from the previous relation and obtain:

$$
J_{\alpha}[f](t) \geq \frac{\alpha^{2}}{d} \frac{\left(\int_{\mathbb{R}^{d}} f^{\alpha}\right)}{\left(\int_{\mathbb{R}^{d}} f^{2 \alpha-1}\right)} I_{\alpha}^{2}(f) \geq \frac{1}{d(1+d(\alpha-1))} I_{\alpha}^{2}(f) .
$$

In 1979, Aronson and Bénilan obtained a second-order differential inequality of the form [20]:

$$
\sum_{i=1}^{d} \frac{\partial}{\partial x_{i}}\left(\alpha f^{\alpha-2} \frac{\partial f}{\partial x_{i}}\right)=\Delta\left(\frac{\alpha}{\alpha-1} f^{\alpha-1}\right) \geq-\frac{d}{(d(\alpha-1)+2) t}, \quad \alpha>\alpha_{c}:=1-\frac{2}{d},
$$

which applies to all positive smooth solutions of the porous medium equation defined on the whole Euclidean space. For general solutions and $d=1$ the restriction is $\alpha>0$. In 1986, Li-Yau studied a heat type flow [21] on complete Riemannian manifolds $(M, g)$ with a nonnegative Ricci scalar, and for any positive function $f$ on $\mathrm{M}$ and any $t>0$, they arrived at the following sharp lower bound:

$$
\Delta(\ln f) \geq-\frac{d}{2 t}
$$

pointwisely, where $\Delta$ is the Laplace-Beltrami operator on $M$. An extension of the Aronson and Bénilan estimate to the PME flow for all $\alpha>1$ and the FDE flow for $\alpha \in\left(\alpha_{c}, 1\right)$ on complete Riemannian manifolds with Ricci scalar bounded from below was given in [22].

In our case, these bounds change, because our pdf differs from the solution of the PME derived from the gradient flow of the functional [23]:

$$
E[f](t)=\frac{1}{1-\alpha} \int_{\mathbb{R}^{d}} f^{\alpha}(x, t) d x .
$$




\section{Conclusion}

In this article, we have proven that the pdfs that maximize Rényi's entropy under the conditions of finite variance and of zero or non-zero mean are given by a one-parameter family of functions, which belong to $S\left(\mathbb{R}^{d}\right)$ for $\alpha<1$ and to $C_{c}^{l}\left(\mathbb{R}^{d}\right)$ for $\alpha>1$. This one-parameter family of functions is a global maximum of the entropy and satisfies the non-linear diffusion Equation (45). The $\mathcal{L}_{\infty}$ norms of these solutions when compared to the corresponding ones derived from the fast and porous medium diffusion equation initial value problem (38) appear to behave differently, as seen in Figures 1 and 2.

If one considers finite even moments of the random variable $X$, as constraints, and tries to solve the corresponding maximization problem, then the $S(\mathbb{R})$ solution exists whenever $\hat{f}^{\alpha-1}$ is a complete polynomial of even degree or, equivalently, when all of the coefficients $\tilde{\lambda}_{2 k+1}$ vanish. In those cases, there exists the possibility not to have interception points with the $\mathrm{x}$-axis, since the roots come into conjugate complex pairs. The compactly-supported solution, under certain conditions, can always be determined.

The concavity of the entropy power holds whenever the second time derivative of the entropy varies according to (49) or the function $f^{\alpha}-C f^{2 \alpha-1}$ belongs to $L_{1}\left(\mathbb{R}^{d}\right)$. It would be more appealing to have a deeper understanding of the origin of the later constraint, which, at this stage, seems to be a requirement for consistency.

\section{Acknowledgements}

The author would like to thank Jiannis K. Pachos for the fruitful discussions related to this project, the Department of Physics and Astronomy of the University of Leeds for its hospitality during my visit (May-July 2015) and the anonymous reviewers for their constructive comments and suggestions, which improved the quality of this article.

\section{Conflicts of Interest}

The author declares no conflict of interest.

\section{Appendix A}

Using the integral Formulas (3.241.4) and (3.251.1) of [18], one can prove the following ones, used in Section 3,

$$
\int_{0}^{\infty} x^{\mu-1}\left(1+x^{2}\right)^{\nu-1} d x=\frac{1}{2} \int_{0}^{1} u^{\frac{\mu}{2}-1}(1-u)^{-\nu-\frac{\mu}{2}}=\frac{1}{2} B\left(\frac{\mu}{2}, 1-\nu-\frac{\mu}{2}\right),
$$

where we made the change of variable $u=x^{2} /\left(1+x^{2}\right)$.

$$
\int_{\mathbb{S}_{d-1}} \int_{0}^{\infty} \frac{r^{d-1}}{\left(C(\alpha)+g(\alpha) r^{2}\right)^{\lambda(\alpha)}} d r d S=\left|\mathbb{S}_{d-1}\right| \frac{C^{\frac{d}{2}-\lambda}}{2 g^{\frac{d}{2}}} B\left(\frac{d}{2}, \lambda-\frac{d}{2}\right) \text { where } C, g, \lambda>0, d<2 \lambda
$$

and $\left|\mathbb{S}_{d-1}\right|=\frac{2 \pi^{\frac{d}{2}}}{\Gamma\left(\frac{d}{2}\right)}$ is the volume of the unit ball and $B(x, y)=\Gamma(x) \Gamma(y) / \Gamma(x+y)$ is the Euler's beta function. The constraint $d<2 \lambda$ for $\lambda=1 /(1-\alpha)$ allows only values:

$$
\alpha_{c_{0}}=1-\frac{2}{d}<\alpha<1 \quad \text { for } \quad d \geq 3
$$


while $\alpha_{c_{0}}=0$ for $d=1,2$.

$$
\int_{\mathbb{S}_{d-1}} \int_{0}^{\infty} \frac{r^{d+1}}{\left(C(\alpha)+g(\alpha) r^{2}\right)^{\lambda(\alpha)}} d r d S=\left|\mathbb{S}_{d-1}\right| \frac{C^{\frac{d}{2}+1-\lambda}}{2 g^{1+\frac{d}{2}}} B\left(\frac{d}{2}+1, \lambda-\frac{d}{2}-1\right) \text { where } d+2<2 \lambda
$$

The constraint between $d, \lambda$ becomes: $\alpha_{c_{2}}=1-\frac{2}{d+2}<\alpha<1 \quad$ for $\quad d \geq 1$.

The last formula is:

$$
\int_{\mathbb{S}_{d-1}} \int_{0}^{\infty} r^{d-1}\left(C(\alpha)-g(\alpha) r^{2}\right)_{+}^{k(\alpha)} d r d S=\left|\mathbb{S}_{d-1}\right| \frac{1}{2} g(\alpha)^{-\frac{d}{2}} C(\alpha)^{\frac{d}{2}+k(\alpha)} B\left(\frac{d}{2}, k(\alpha)+1\right) \text { if } k(\alpha)>0 .
$$

Formulas (A.2) and (A.3) are direct consequences of (3.241.4), (3.251.1) and (A.1). Another integral formula used in Section 4 is (3.252.6) of [18].

Identities involving the Euler's beta function:

$$
\lim _{\rho \rightarrow \infty}\left(\rho^{\frac{s}{2}} B\left(\frac{s}{2}, \rho-\frac{s}{2}\right)\right)=\Gamma\left(\frac{s}{2}\right)
$$

Proof of (A.6): The large asymptotic expansion of the ratio of gamma functions [24] is given by:

$$
\frac{\Gamma(\rho+a)}{\Gamma(\rho+b)}=\rho^{a-b}\left(1+\frac{(a-b)(a+b-1)}{\rho}+O\left(\rho^{-2}\right)\right) .
$$

Substituting the values $a=-s / 2, b=0$, in the previous expression and taking the limit $\rho \rightarrow \infty$, we recover the desired result.

$$
\begin{aligned}
& d \frac{B\left(\frac{d}{2}, \frac{1}{1-\alpha}-\frac{d}{2}\right)}{B\left(\frac{d}{2}, \frac{\alpha}{1-\alpha}-\frac{d}{2}\right)}+(d-2) \frac{B\left(1+\frac{d}{2}, \frac{\alpha}{1-\alpha}-\frac{d}{2}\right)}{B\left(\frac{d}{2}, \frac{\alpha}{1-\alpha}-\frac{d}{2}\right)}=\frac{d}{\alpha}(2 \alpha-1) \\
& d \frac{B\left(\frac{d}{2}, \frac{\alpha}{\alpha-1}\right)}{B\left(\frac{d}{2}, \frac{\alpha}{\alpha-1}+1\right)}-(d+2) \frac{B\left(1+\frac{d}{2}, \frac{1}{\alpha-1}+1\right)}{B\left(\frac{d}{2}, \frac{\alpha}{\alpha-1}+1\right)}=\frac{d}{\alpha} .
\end{aligned}
$$

Proof of (A.8): These are direct consequences of the definition of the Euler's beta function and the gamma's function property $\Gamma(x+1)=x \Gamma(x)$.

$$
\begin{aligned}
B\left(k+\frac{1}{2}, n-k-\frac{1}{2}\right) & =(2 k-1) ! ! \frac{(2 n-2 k-3) ! !}{(2 n-2) ! !} \pi, \quad k \in \mathbb{Z}^{+} \\
B\left(\frac{1}{2}, n+1\right) & =2^{2 n+1} B(n+1, n+1) .
\end{aligned}
$$

Proof of (A.9): Use the doubling formula for gamma functions and $\Gamma(n)$ in terms of the double factorial:

$$
\Gamma\left(n+\frac{1}{2}\right)=\frac{\sqrt{\pi}}{2^{n}}(2 n-1) ! !, \quad \Gamma(n)=\frac{(2 n-2) ! !}{2^{n-1}} .
$$

Proposition 7.1. The function $\Gamma(x) / \Gamma(x+c)$ with $x, c \in \mathbb{R}^{+}$is decreasing in $x$.

Proof of (7.1): It is enough to show that:

$$
\frac{d}{d x} \ln \left(\frac{\Gamma(x)}{\Gamma(x+c)}\right)=\psi(x)-\psi(x+c)<0 .
$$


A straightforward computation gives:

$$
-\ln \left(1+\frac{c}{x}\right)-\frac{c}{x(x+c)} \leq \psi(x)-\psi(x+c) \leq-\ln \left(1+\frac{c}{x}\right)-\frac{c}{2 x(x+c)}<0
$$

by using the inequality: $\ln x-\frac{1}{x} \leq \psi(x) \leq \ln x-\frac{1}{2 x}$.

\section{References}

1. Horodecki, R.; Horodecki, P.; Horodecki, M.; Horodecki, K. Quantum entanglement. Rev. Mod. Phys. 2009, 81, 865-942.

2. Evangelisti, S. Quantum Correlations in Field Theory and Integrable Systems; Minkowski Institute Press: Montreal, QC, Canada, 2013.

3. Geman, D.; Jedynak, B. An active testing model for tracking roads in satellite images. IEEE Trans. Pattern Anal. Machine Intell. 1996, 1, 10-17.

4. Jenssen, R.; Hild, K.E.; Erdogmus, D.; Principe, J.C.; Eltoft, T. Clustering using Renyi’s Entropy. In Proceedings of the International Joint Conference on Neural Networks, Portland, OR, USA, 20-24 July 2003; pp. 523-528.

5. Bennett, C.H.; Brassard, G.; Crépeau, C.; Maurer, U.M. Generalized privacy amplification. IEEE Trans. Inf. Theory 1995, 41, 1915-1923.

6. Sahoo, P.; Wilkins, V.; Yeager, J. Threshold selection using Reny'is entropy. Pattern Recognit. 1997, 30, 71-84.

7. Sankur, B.; Sezgin, M. Image thresholding techniques: A survey over categories. Pattern Recognit. 2001, 34, 1573-1607.

8. Johnson, O.; Vignat, C. Some results concerning maximum Rényi entropy distributions. Annales de l'Institut Henri Poincare (B) 2007, 43, 339-351.

9. Barenblatt, G.I. On some unsteady motions of a liquid and gas in a porous medium. Prikl. Mat. Mec. 1952, 16, 67-78.

10. Barenblatt, G.I. Scaling, Self-Similarity and Intermediate Asymptotics; Cambridge University Press: Cambridge, UK, 1996.

11. Zeld'dovich, Y.B.; Kompaneets, A.S. Towards a theory of heat conduction with thermal conductivity depending on the temperature. In Collection of Papers Dedicated to 70th Birthday of Academician A. F. Ioffe; Izd. Akad. Nauk SSSR: Moscow, Russia, 1950; pp. 61-71.

12. Villani, C. A short proof of the "concavity of entropy power". IEEE Trans. Inf. Theory 2000, 46, 1695-1696.

13. Savaré, G.; Toscani, G. The concavity of Rényi entropy power. IEEE Trans. Inf. Theory 2014, 60, 2687-2693.

14. Liberzon, D. Calculus of Variations and Optimal Control Theory; Princeton University Press: Princeton, NJ, USA, 2012.

15. Havrda, M.; Charvát, F. Quantification method of classification processes: Concept of structural $\alpha$-entropy. Kybernetika 1967, 3, 30-35.

16. Tsallis, C. Possible generalizations of Boltzmann and Gibbs statistics. J. Stat. Phys. 1988, 52, 479-487. 
17. Lutwak, E.; Yang, D.; Zhang, G. Cramér-Rao and moment-entropy inequalities for Rényi entropy and generalized Fisher information. IEEE Trans. Inf. Theory 2005, 51, 473-478.

18. Gradshteyn, I.S.; Ryzhik, I.M. Table of Integrals, Series, and Products; Academic Press: Boston, MA, USA, 1994.

19. Vázquez, J.L. The Porous Medium Equation: Mathematical Theory; Oxford University Press: Oxford, UK, 2007.

20. Aronson, D.G.; Bénilan, P. Régularité des solutions de l'équation des milieux poreux dans $\mathbb{R}^{n}$. C. R. Acad. Sci. Paris. Sér. 1979, A-B 288, 103-105.

21. Li, P.; Yau, S.T. On the parabolic kernel of the Schrödinger operator. Acta Math. 1986, 56, 153-201.

22. Lu, P.; Ni, N.; Vázquez, L.J.; Villani, C. Local Aronson-Bénilan estimates and entropy formulae for porous medium and fast diffusion equations on manifolds. J. Math. Pures Appl. 2009, 91, 1-19.

23. Otto, F. The geometry of dissipative evolution equations: The porous medium equation. Commun. Partial Differ. Equ. 2001, 26, 101-174.

24. Tricomi, F.G.; Erdélyi, A. The asymptotic expansion of a ratio of gamma functions. Pacific J. Math. 1951, 1, 133-142.

(c) 2015 by the author; licensee MDPI, Basel, Switzerland. This article is an open access article distributed under the terms and conditions of the Creative Commons Attribution license (http://creativecommons.org/licenses/by/4.0/). 